\title{
Tracking of Neoclassical Tearing Modes in TCV using the Electron Cyclotron Emission diagnostics in quasi-in-line configuration
}

\author{
N. Rispoli ${ }^{\mathrm{a}, *}$, C. Sozzi ${ }^{\mathrm{a}}$, L. Figini ${ }^{\mathrm{a}}$, D. Micheletti ${ }^{\mathrm{a}}$, C. Galperti ${ }^{\mathrm{b}}$, M. Fontana $^{\mathrm{b}}$, E. Alessi $^{\mathrm{a}}$, S. Coda $^{\mathrm{b}}$, S. Garavaglia $^{\mathrm{a}}, \mathrm{T}^{\mathrm{T}}$ \\ Goodman $^{\mathrm{b}}$, M. Kong ${ }^{\mathrm{b}}$, M. Maraschek ${ }^{\mathrm{c}}$, A. Moro ${ }^{\mathrm{a}}$, L. Porte ${ }^{\mathrm{b}}$, O. Sauter ${ }^{\mathrm{b}}$, U. Sheikh ${ }^{\mathrm{b}}$, D. Testa ${ }^{\mathrm{b}}$, EUROfusion MST1 \\ Team $^{1}$, and TCV team ${ }^{2}$ \\ ${ }^{a}$ Istituto di Fisica del Plasma "Piero Caldirola" - CNR, Milano, Italy \\ ${ }^{b}$ Ecole Polytechnique Fdrale de Lausanne (EPFL), Swiss Plasma Center (SPC), Lausanne, Switzerland \\ ${ }^{c}$ Max Planck Institute for Plasma Physics, Garching, Germany
}

\begin{abstract}
An important goal of the control system in a tokamak is the suppression of magneto-hydrodynamic (MHD) instabilities with low $\mathrm{m}, \mathrm{n}$ (poloidal and toroidal mode numbers), which can influence the confinement time of energy and particles and possibly lead to plasma disruption. These instabilities, which appear as rotating magnetic islands, can be reduced or completely suppressed by a current driven by electron cyclotron waves (ECW) accurately located within the island. A fundamental requisite for this control technique is the ability to identify the island parameters (amplitude and radial position) and to vary accordingly the ECW deposition location. Here we describe a control scheme of the steering mirror of the ECW source based on the real-time tracking of the island radial position realized using only the electron cyclotron emission (ECE) diagnostics in quasi-in-line configuration, i.e. with toroidal anti-parallel propagation of the ECW and ECE beams. The successful experimental proof of principle of this scheme, tested on the TCV tokamak, is here reported.
\end{abstract}

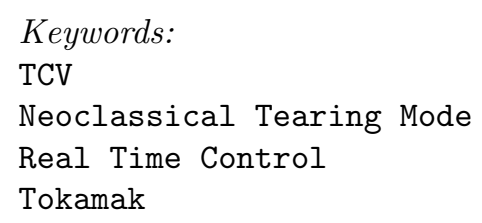

\section{Introduction}

One of the limiting factors of the tokamak performance arises from the magnetic-hydrodynamic instabilities. They present themselves as rotating islands at colder tempera5 tures that reduce the plasma confinement and, depending on their size and growth rate, can lead to disruptions. The reduction or suppression of magneto-hydrodynamic instabilities, in particular of Neoclassical Tearing Modes $(N T M s)$, can be performed through localized current 10 driven by Electron Cyclotron Wave $(E C W)$. This is a challenging control task that requires: the detection of spatial position of the instability; steering of the mirror in the correct position and the triggering of the electron cyclotron $E C W$ sources. To achieve this goal a few computational steps are needed, such as magnetic equilibrium reconstruction, $E C W$ beam tracing, correct time phasing and angular steering of the $E C W$ injection. To successfully apply $E C$ waves for $N T M$ control it is important that the island is detected as early as possible and that its

\footnotetext{
* Corresponding author

Email address: natale.rispoli@ifp.cnr.it (N. Rispoli)

${ }^{1}$ See the author list of H. Meyer et al 2017 Nucl. Fusion $57102014{ }^{40}$

${ }^{2}$ See the author list of S. Coda et al 2017 Nucl. Fusion 57102011
}

20 localizzation is identified with an accuracy equal or even lower than the radial extension of the $E C W$ beam width.

A closed loop control scheme using quasi-in-line configuration of $E C E$ and $E C W$ beam allows real time tracking and NTMs suppression without the need of knowing a priori the exact mirror steering angle, without precise magnetic equilibrium reconstruction and without beam tracing computation. The proof of principle of this control scheme was demostrate on $T C V$, using different values of poloidal and toroidal shift between $E C W$ and $E C E$, and has described in this paper. The toroidal injection angle is kept constant and equal for both ECE and ECW, while the poloidal aiming of the ECW antenna keeps into account the poloidal shift.

A brief description of $T C V$ control system and NTM control scheme are presented in section 2, The detection algoritm $(D A)$ is described in section 3 . The main results are reported in section 4 .

\section{TCV System and NTM Control scheme}

$T C V$ is a medium sized tokamak with major radius of $0.88 \mathrm{~m}$, minor radius of $0.25 \mathrm{~m}$ and maximum toroidal field of $1.5 T([1])$. A distributed digital RT control system, 
with highly modular structure, and connected with a wide range of plasma diagnostics permit to develop control algorithms independently and to be integrated into the multicontroller environment.

The control scheme, realized in such environment, have been tested using ECE radiometer (2]) with $L 3$ launcher 75 transmission line and $E C W$ with launcher $L 1$ or $L 2$, see Figure 2, $L 1$ and $L 3$ are at same toroidal location with 50 a poloidal shift, while $L 2$ is at same poloidal shift but in a different toroidal sector. All launchers ( $L 1, L 2$ and $L 3)$ are identical antenna systems based on steerable mirrors. Figure 1 shows the experimental configuration (left) and the simplified control scheme (right).

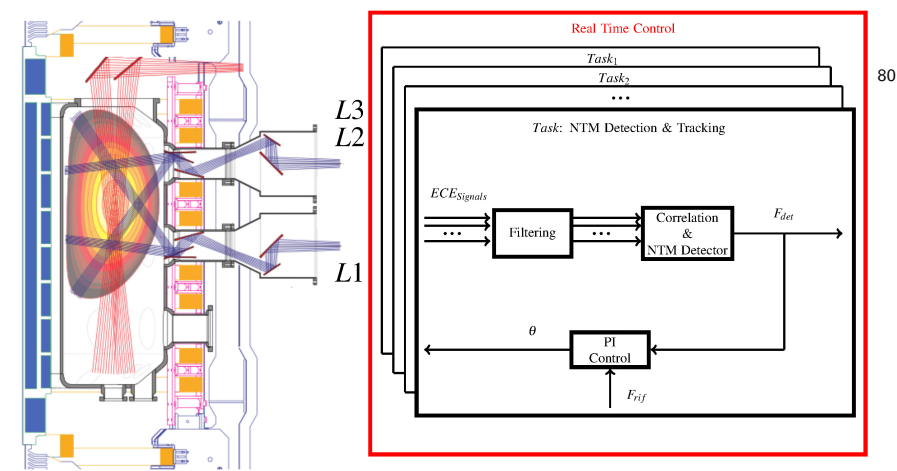

Figure 1: Simplified scheme of NTM detection and mirror control

The ECE system has 24 channels, divided in: 1) 12 channels on low field side $(L F S) 66.15 \leq f \leq 82.15 \mathrm{GHz}$; 2) 12 channel on high field side (HFS) $85.48 \leq f \leq 101.48$ $\mathrm{GHz}$. Signals, collected through this system, are used for detect the plasma frequency location of NTM instability.

The detected frequency $\left(F_{i s l}\right)$ is used by proportionalintegral $(P I)$ control to move $L 3$ mirror at angle corresponding to target frequency $\left(F_{\text {rif }}\right)$, which is the angle where the frequency detected by $E C E$ radiometer is equal to $E C W$ frequency $(82.7 G H z)$. The mirror on $L 1$ or $L 2$ 65 was moved accordingly to $L 3$ and the $E C W$ power is activated when the target frequency is reached.

\section{Detection Algorithms}

The algorithm by Berrino et al. ([3]), that uses only ECE signals, has been used to detect the island location 70 in the frequency space. Correlating adjacent frequency channels signals, the algorithm detects the phase opposition between ECE signals on opposite sides of frequency 95 corresponding to the rational flux surface. It can be divided into three different calculation steps.

Defined $E C E_{i}(k)$ the signal measured by the i-th ECE channel at frequency $f_{i}$ and at time $k$, in the first step the oscillation information is extracted from signals using a band pass filter and the results $\left(\delta E C E_{i}\right)$ is diveded by the root mean square value:

$$
{\overline{\delta E C E_{i}}}_{i}(k)=\frac{\delta E C E_{i}(k)}{\sqrt{\frac{1}{N_{r m s}} \sum_{j=0}^{N_{r m s}} \delta E C E_{i}(k-j)}}
$$

The correlation between adjacent ECE channels is calculated in the second step as:

$$
C_{i}=\frac{1}{N_{c}} \sum_{j=0}^{N_{c}}{\overline{\delta E C E_{i}}}_{i}(k-j) *{\overline{\delta E C E_{i+1}}}_{(k-j)}
$$

thus obtaining a set of $N_{E C E}$ correlation functions $C_{i}$, $N_{E C E}$ being the number of channels of the diagnostics.

In the third and final step, the effort is in identify the presence and location of the island $\left(\bar{f}_{i s l}\right)$. The island presence can be identified when the second derivative of correlation functions $\left(C_{i}\right)$ exceeds a fixed threshold $(s)$. The fixed threshold $s$ avoid false detections due to the measurement noise.

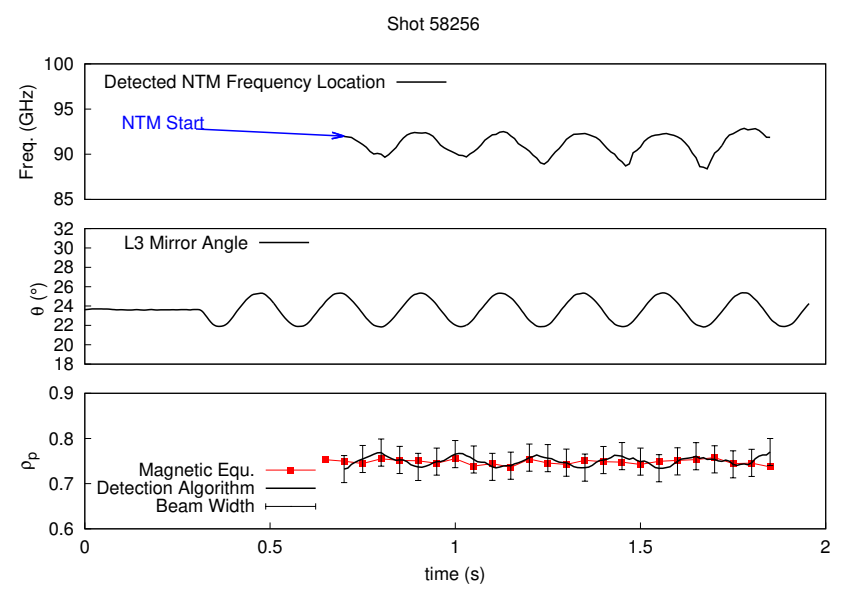

Figure 2: Frequency detected by DA algorithm (top), preprogrammed mirror steering (center) and minor radius of the emission region vs time (bottom)

Figure 2 shows the results of the setup procedure, with preprogrammed mirror movement and NTM at $q=2$ rational surface, used to set the threshold level. A value $s=0.06 / \Delta f^{2}$ is found to yield no false detections, where $\Delta f=1.46 \mathrm{GHz}$ is the ECE channels spacing. In Figure 2 90 (bottom) the comparison between the minor radius of the rational surface identified by the algorithm and the one reconstructed offline by the magnetic equilibrium shows that the detected instability location is centered on the rational surface with an accuracy smaller than the $E C E$ beam width.

\section{Results}

The control scheme was tested on NTM instabilities on the rational surface $q=2$. Due to technical problems in the operation and to the limited signal/noise of the $L F S$, 
nly the $H F S$ radiometer channels have been used. Under these conditions the minimum attainable frequency was 88 $G H z$, corresponding to $80.5 \mathrm{GHZ}$ on $L F S$. Therefore, since it is not possible to reach the $E C W$ frequency, the triggering condition for the gyrotron can not bet reached 105 unless of relaxing the threshold on the target frequency. This was successfully done on Shot 58680 .

Both L1 (Shot 58680,59252) and L2 (Shot 59245) launchers have been used with similar results, only the results for the $L 1$ launcher configuration are shown here.

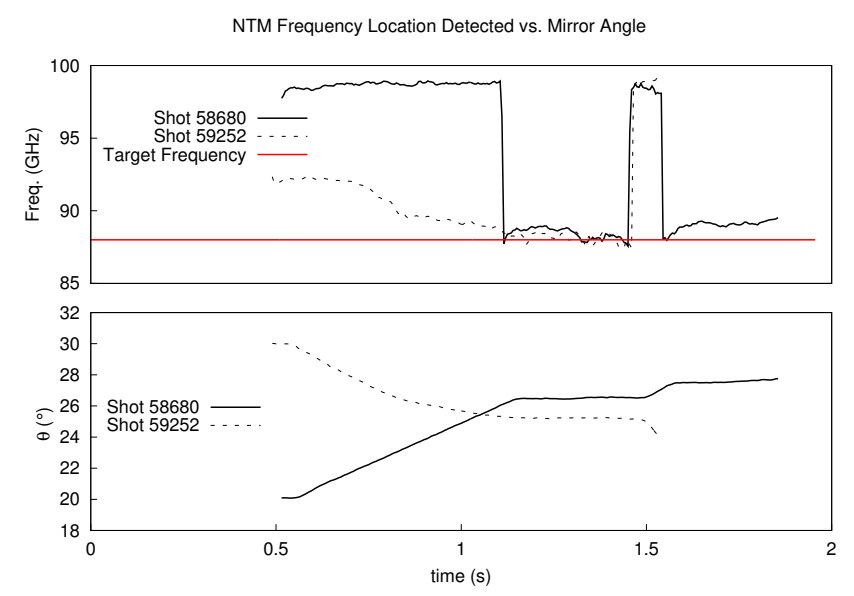

Figure 3: NTM frequency detection (top) and mirror steering (bottom) for TCV shot 58680 (solid line) and shot 59252 (dashed line).

Figure 3 (top) shows the frequency detection results and ${ }^{130}$ mirror steering (bottom) for two TCV shots, with mirrors starting position $\theta=20^{\circ}$ (solid curve) and $\theta=30^{\circ}$ (dashed line). The minor radius of the emission region as a function of the $L 3$ angle and of the frequency, obtained in postprocessing with the SPECE code([4]), is shown in figures ${ }^{135}$ 4 (shot 58680) and 5 (shot 59252).

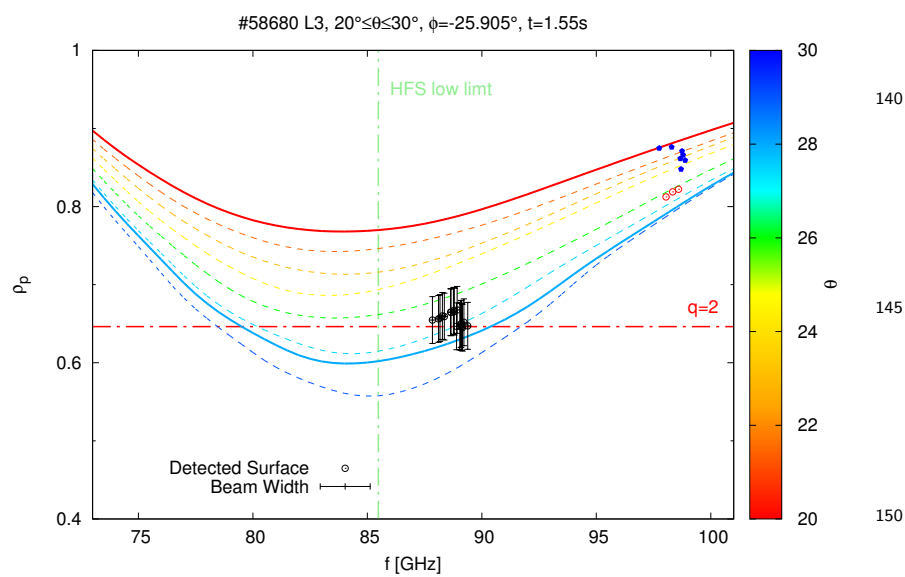

Figure 4: Minor radius of the emission region for Shot 58680 as function of $L 3$ angle and frequency

Experimental results show the $D A$ capability to detect the NTM instability, with and error comparable with beam width, when $\theta>25^{\circ}$. It is possible to notice the 155

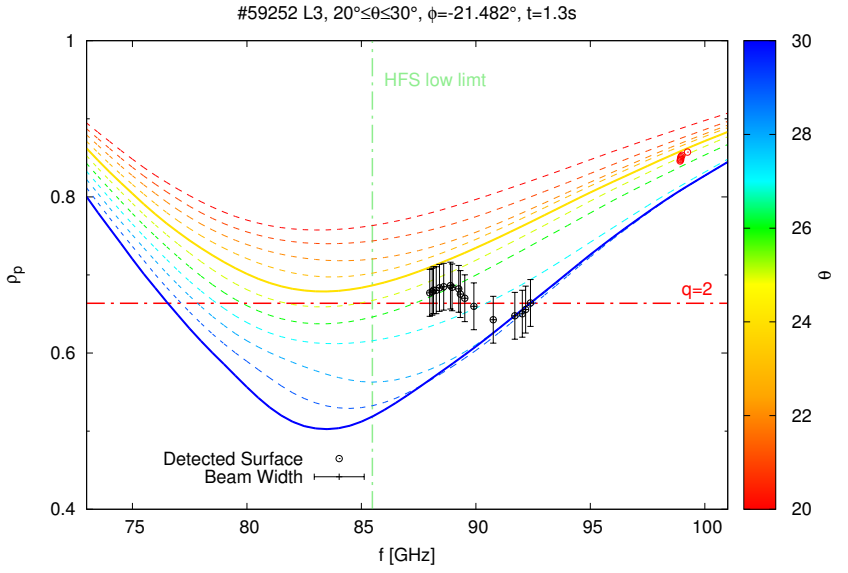

Figure 5: Minor radius of the emission region for Shot 59252 as function of $L 3$ angle and frequency

loss of precision in detection for $\theta<25^{\circ}$ when there are no channels on opposite sides of instability (full points at the top right of the Figure 4). Under these conditions the algorithm detects the oscillations on the temperature signal and therefore the presence of an NTM instability, but it is not able to identify the exact spatial position. Moreover when the line of sight is tangent to the instability rational surface, at $25^{\circ}<\theta<26^{\circ}$, a displacement of the plasma can lead to an error in the NTM spatial localization (empty points at the top right of the Figure 4 and 5). Similar conditions have already been discussed in [5] for the study of quasi-in-line configurations in ITER. These limitations can be solved or reduced by new DA exploiting the signals of all the ECE radiometer channels.

The experimental results support the observation that for starting positin of the mirror located at the plasma edge (corresponding to $\theta=20^{\circ}$ ), despite the loss of spatial detection, the PI control can move the mirror to reach the target position and recover any loss of detection. Conversely, for starting position at the plasma center $\left(\theta=30^{\circ}\right)$ the NTM tracking is more precise, but the controller is not able to recover stability when a loss of spatial detection occurs. This behavior largely depends on the DA setup which require further optimization.

Figure 6 shows the difference between $L 1$ and $L 3$ minor radius of the emission region as a function of the frequency and the mirrors steering angle for Shot 58680, only for the points in the zone of successfull detection. These points show that the spatial location of $E C W$ is always smaller than the beam width. It can be noticed that using LFS ECE channels it would be possible to reach the desired frequency of $82.7 \mathrm{GHz}$ with a better accuracy.

\section{Conclusions}

A real time closed loop control scheme to track and suppress $N T M$ instabilities was realized and implemented in $T C V$. The proof of principle of this scheme was done by 


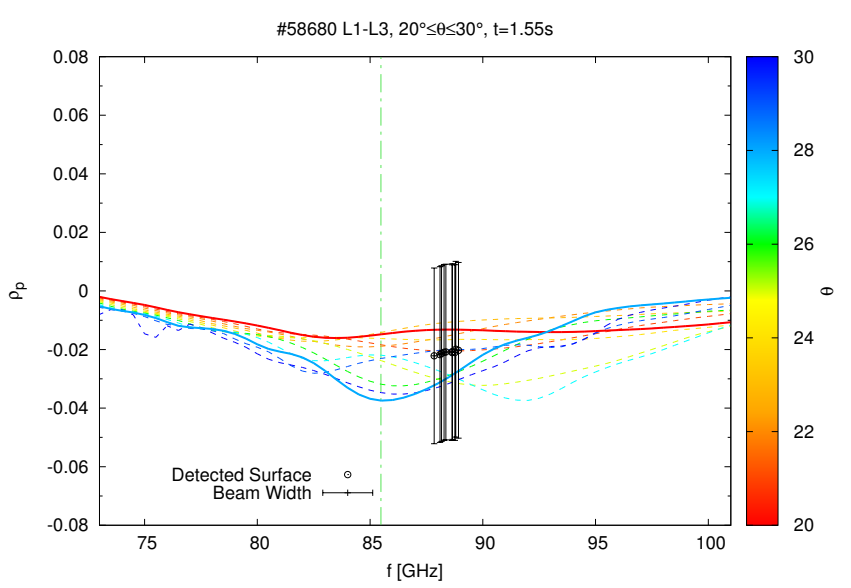

Figure 6: Difference between $L 1$ and $L 3$ minor radius of the emission region for Shot 58680 as function of mirrors angle and frequency

mimicking quasi-in-line configuration of $E C E$ and $E C W$ beam.

Results show that the control algorithm was able to track the $N T M$ instability and to aim the $E C W$ at the $N T M$ instability position with an accuracy smaller than the beam width. However, using only the HFS ECE channels, the control scheme was not able to follow the $N T M$ in all possible spatial positions, in particular it was not possible to steer the mirror at the desired target position of $82.7 \mathrm{GHz}$. This is mainly due to present limitation of the radiometer and this can be partially overcome introducing new features in the detection algorihm.

Based on the successfull preliminary results obtained here, a new detection scheme using all ECE channels with a more complex control, which includes an initial preprogrammed scan phase over all mirror steering angle, is being developed and will be tested in the next experimental campaign.

\section{References}

[1] S. C. at all., Nuclear Fusion 57 (2017) 102011. URL: http:// stacks . iop.org/0029-5515/57/i=10/a=102011

[2] M. Fontana, L. Porte, P. Marmillod, EPJ Web Conf. 147 (2017) 02005. URL: https://doi.org/10.1051/epjconf/201714702005 doi 10.1051/epjconf/201714702005

[3] J. Berrino, E. Lazzaro, S. Cirant, G. D'Antona, F. Gandini, E. Minardi, G. Granucci, Nuclear Fusion 45 (2005) 1350. URL: http://stacks.iop.org/0029-5515/45/i=11/a=016.

[4] D. Farina, L. Figini, P. Platania, C. Sozzi, AIP Conference Proceedings 988 (2008) 128-131. URL: http://scitation.aip.org/

185 content/aip/proceeding/aipcp/10.1063/1.2905053 doi/http: //dx.doi.org/10.1063/1.2905053

[5] L. Figini, D. Farina, D. Micheletti, N. Rispoli, C. Sozzi, Proceedings of 43rd EPS Conference on Plasma Physics (2016) 945-948.

\section{Acknowledgment}

This work has been carried out within the framework of the EUROfusion Consortium and has received funding from the Euratom research and training programme 20142018 under grant agreement No 633053. The views and opinions expressed herein do not necessarily reflect those 5 of the European Commission. 